\title{
Adaptive Backlash Compensation Method Based on Touch State Observation for a Solid Ducted Rocket
}

\author{
An Wang $\mathbb{D},{ }^{1,2}$ Qinghua Zeng $\mathbb{D}^{2},{ }^{2}$ Likun $M a,{ }^{1}$ and Hongfu Wang $\mathbb{D}^{2}$ \\ ${ }^{1}$ College of Aerospace Science and Engineering, National University of Defense Technology, Changsha, Hunan 410073, China \\ ${ }^{2}$ School of Aeronautics and Astronautics, Sun Yat-sen University, Guangzhou, Guangdong 510006, China \\ Correspondence should be addressed to Qinghua Zeng; zqinghua@sysu.edu.cn
}

Received 21 October 2020; Revised 2 November 2020; Accepted 3 November 2020; Published 18 November 2020

Academic Editor: Linda L. Vahala

Copyright ( 2020 An Wang et al. This is an open access article distributed under the Creative Commons Attribution License, which permits unrestricted use, distribution, and reproduction in any medium, provided the original work is properly cited.

\begin{abstract}
A hysteresis model was built to describe the backlash of the flow regulator in a solid ducted rocket, and its influence on the engine control was also analyzed in this study. An adaptive backlash compensation method was proposed under two challenges: limited information and backlash state variation caused by the harsh environment in the gas generator. The touch state is designed and its observation is used to get the state of backlash, and a compensation control method using the existing information was carried out combined with the motion intention. This method greatly shortened the time during the transition and reduced the hysteresis effect on the control system. Furthermore, the compensation method is improved and acquires a self-learning ability, the compensation parameter changes adaptively during the process of flow regulation, and it is able to meet the challenge of an unknown and variable state of backlash. Finally, the validation of the compensation method was carried out with two simulations.
\end{abstract}

\section{Introduction}

A solid ducted rocket is a propulsion device developed based on solid rocket and ramjet technology. They exhibit the advantages of high specific impulse, long range, small size, low weight, and compact structure. Hence, they are optimal propulsion devices for new-generation aircraft [1-4]. The gas flow control system for a solid ducted rocket is generally designed based on the predetermined rocket trajectory in the early days, usually realized in the form of a prefabricated propellant grain structure. Hence, the performance of a ducted rocket decreases rapidly once the flight condition deviates from the design condition, and consequently, the trajectory of an aircraft using this engine becomes severely restricted. Due to the presence of a variety of disturbances during real flight, active control technology for gas flow regulation has become the key focus of ducted rocket research.

Active control technology of a ducted rocket is closely related to the flow regulation device; it is an important part of the engine control system and plays the role of an actuator in the control system. As an important component of a solid ducted rocket, the development of flow regulation is a key step to the active control of ducted rockets; considerable research has been carried out by scholars in various fields.

A flow regulator has been designed to improve the output performance, further broaden the operating envelope, and give full scope to the propellant performance in reference [5]; its intensity and inner flow field were simulated, and the test piece was machined to finish the test. The effect on the engine performance of a pintle valve is studied in reference [6]; through comparison, it has been found that the presence of a pintle valve improves the uniformity of the gas velocity, reduces the outlet velocity, contributes to the diffusion of gas, and improves the premixed combustion and performance of the ducted rocket. In reference [7], the friction generated in gas flow regulation of ducted rockets is considered, a compensation method is discussed to eliminate its effect, and its validation is carried out by a simulation result. The effects of valve velocity on the dynamic response characteristics of a variable-flow ducted rocket motor were analyzed by researching on the numerical simulations in reference [8], and the results indicated that a valve velocity that is either too low or too high is harmful to the performance enhancement of a variable-flow ducted rocket. The valve is a unit directly 
regulating the gas flow, and in order for it to operate, it needs to been driven by a device such as a pneumatic drive, a hydraulic drive, or a motor drive for a flow regulator. At present, the motor drive has the best comprehensive performance: it is safe, reliable, and stable. Furthermore, motor drive devices are compact in structure, which is an advantage to system integration on an aircraft.

As a typical electromechanical system, flow regulator devices face the challenges that are common to many other electromechanical systems; the most typical challenge is the backlash influence. The valve needs to be driven by the motor and transmission mechanism, and during the transfer of force and torque, the backlash is inevitably brought into the process of flow regulation. There is also a lot of research on this typical problem. The study in reference [9] is aimed at identifying a backlash using one-dimensional modeling functions that do not take account of velocity. The study in reference [10] uses a displacement sensor and a position encoder to measure the displacement of the driving part of the mechanical equipment, and it presents a control system for reversing backlash by compensating for high-precision mechanical equipment. A compensation method based on global back-stepping sliding mode control is proposed in reference [11]; it not only compensates for the backlash in a naval gun servo system but it also weakens the chatter of the driving torque and the load speed during the commutation period. To achieve high precision even with transmission mechanisms, the study in reference [12] proposes a precise joint torque control method with backlash compensation by using load-side encoder information. An adaptive fuzzy tracking control for a system with an unknown nonlinear function and an unmeasurable state is carried out in reference [13]. Fuzzy logic systems are used to approximate the unknown nonlinear functions, and a fuzzy observer is designed to estimate the immeasurable states. An adaptive tracking control scheme is proposed for a class of switched nonlinear systems with state and input unmodeled dynamics in reference [14]. In reference [15], a robust, fault-tolerant $H_{\infty}$ control method for the general actuator fault and perturbation compensation system is proposed.

The research above described a lot of research content and solutions about the backlash. However, the flow regulator in a ducted rocket has its particularity in the process of backlash compensation, and it is mainly reflected in two points. Firstly, as an important part of an aircraft, the components of the ducted rocket have been greatly limited by space and weight; it means that any unnecessary sensors will be saved, and the compensation method using a highprecision sensor in reference $[10,12]$ will not be suitable for use in the flow regulator. On the other hand, if the regulation valve is fully exposed to the harsh environment with the gas generator as the working part, using the displacement sensor is extremely hard. Secondly, among many flow regulation solutions, changing the throat area of the gas generator is the most effective and advanced one, but it is also the most difficult one; the main challenge is to make the throttling valve work effectively for a long time in a complex and harsh working environment. During the process, the backlash state will change under the influence of heat and stress, and the changing state will also bring a challenge to the backlash compensation control. In a word, the backlash compensation method should be proposed considering two challenges: limiting information and changing the state of backlash.

In response to the two challenges mentioned above, touch state and its observation were carried out without adding additional sensors to obtain the state of backlash in this study. Based on the touch state observation, a new backlash compensation method was proposed, and the hysteresis of flow regulation has been solved effectively. And on this basis, the compensation method can be improved and a selflearning ability acquired; the compensation parameter will change adaptively, and it can meet the challenge that the state of backlash is unknown or changing during the process of flow regulation.

The rest of this paper is organized as follows. First, the modeling of flow regulator devices and backlash is given; then, its influence on the system control is analyzed. Then, the definition of touch state and its observation are carried out, and the principle of backlash compensation method based on the touch state is proposed. Finally, the compensation method was discussed in detail and its validation was carried out with simulation in two cases: constant backlash state and changing backlash state.

\section{Modeling of Flow Regulator and Backlash}

2.1. Designing of a Flow Regulator. The flow regulation device is an important actuator of a ducted rocket; with the development of a variable-flow ducted rocket, various types of throttling valves have emerged. Several applicable types of throttling valves are listed in reference [16] as is shown in Figure 1 .

In Figure 1, several types of throttling valves are shown, including the side-inserted body, the spool valve, the guillotine plate, the rotated cam, the rotative plate, and the pintle, and they are compared in terms of the axial space required, the throttling sensitivity and flow effects, the support complexity, the external sealing complexity, and the actuation complexity. The advantages and disadvantages of the different types were analyzed.

For the moment, the design of a flow regulator device needs to consider several factors based on its application needs: spatial layout and weight constraint, thermal protection and airtightness, valve design and material selection, actuator overload standing, risk of solid particles piling up into a block, and processing technology and its realizability.

Based on the application needs, the type of throttling valve designed in this paper differs from the several types mentioned above; it is a gate valve, and its schematic is shown in Figure 2.

The gate valve is driven in the form of rotational motion to change the throat area and regulate the gas flow. The airtightness is easier to realize because of only one drive shaft passing through the gas generator at midstage. The motion direction of the valve is perpendicular to the load force caused by the impact of gas flow, so the actuator does not directly overcome the load and the mechanical load of the system is smaller. The structure of the whole regulating 

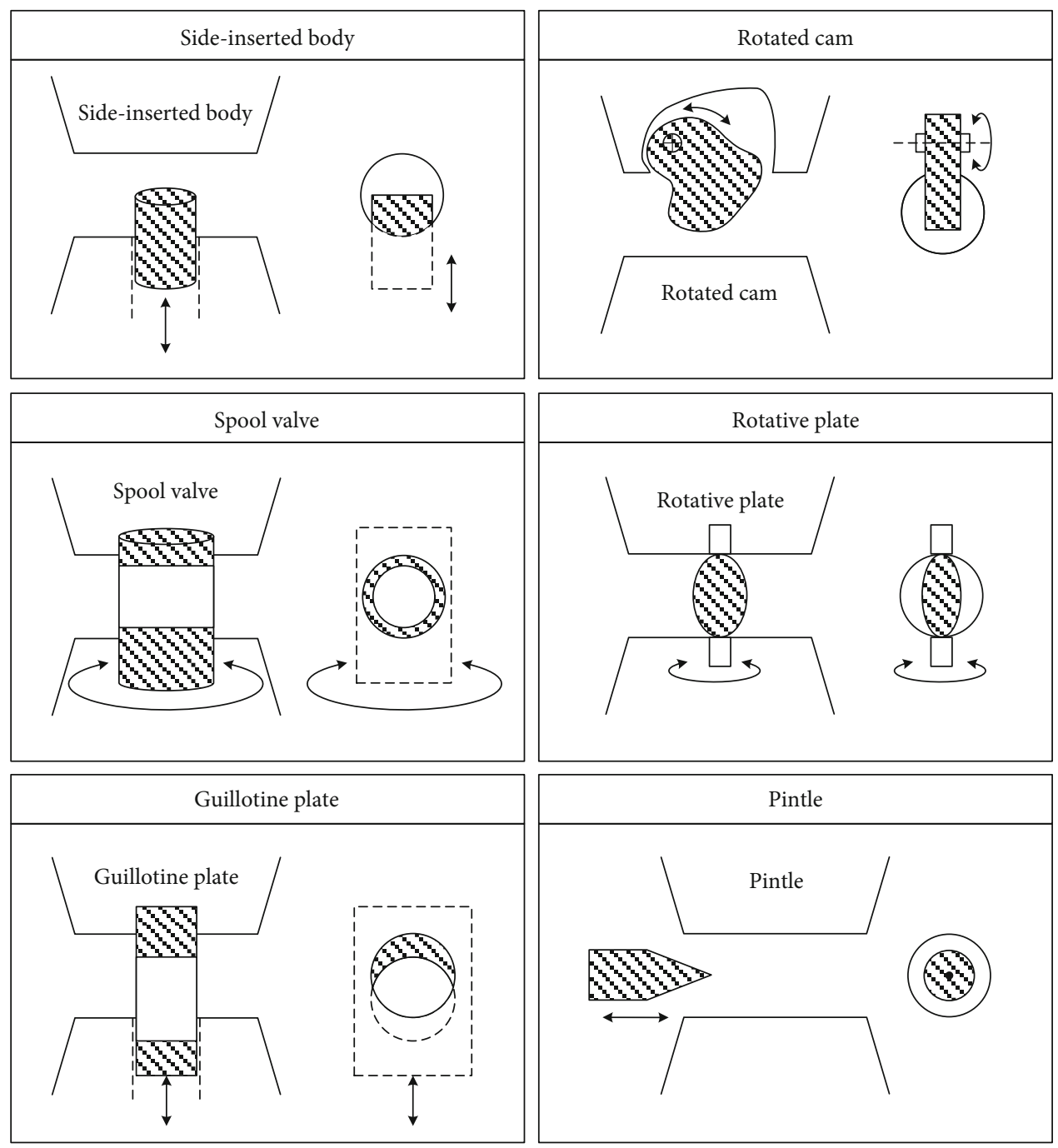

FIgURE 1: Several application types of the throttling valves.

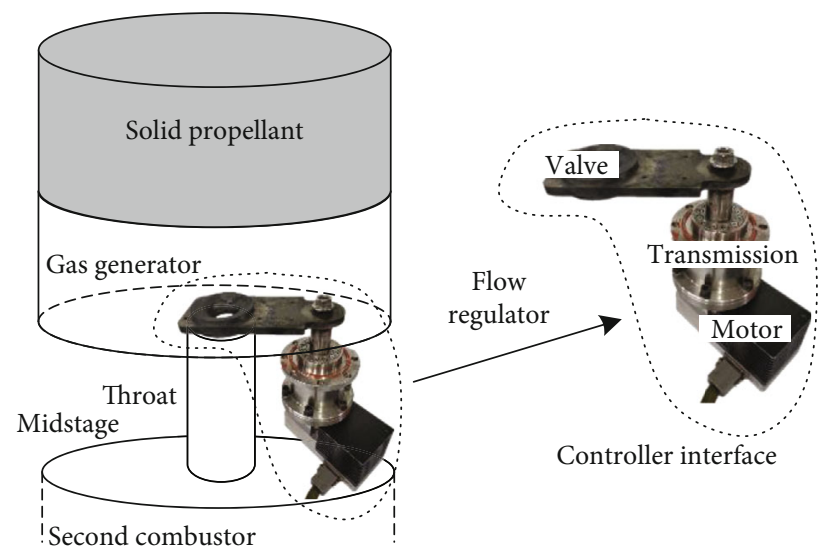

Figure 2: Schematic of the flow regulator using a gate valve.

system is simple and compact, and it occupies a small space. However, the whole gate valve and part of the driveshaft are exposed to the gas flow with high temperature and high speed, and the thermal load and ablation are a severe chal- lenge. The mechanical interface of the valve and the driveshaft is also exposed in such a harsh environment, and it will have an effect on gas flow regulation; the details are discussed in Subsections 2.2 and 2.3.

The relation between the valve angle and the throat area is shown in Figure 3, and the throat area increases with the valve angle monotonically.

2.2. Modeling of Backlash. In a general electromechanical system, the active unit is often not the drive unit. Transmission devices are required to transfer the driving forces and torque, and in the process of transfer, the backlash is brought into the flow regulation system more or less. There are various existing forms of backlash in a gas generator: clearance of assembly accuracy, designing backlash for thermal expansion, backlash in the motor, backlash of stress, and so on. In a word, backlash exists in different units with different causes, and it is complicated to describe the backlash in a gas generator.

For ease of description and analysis, the regulation devices are unified into a whole system, taking the motor 

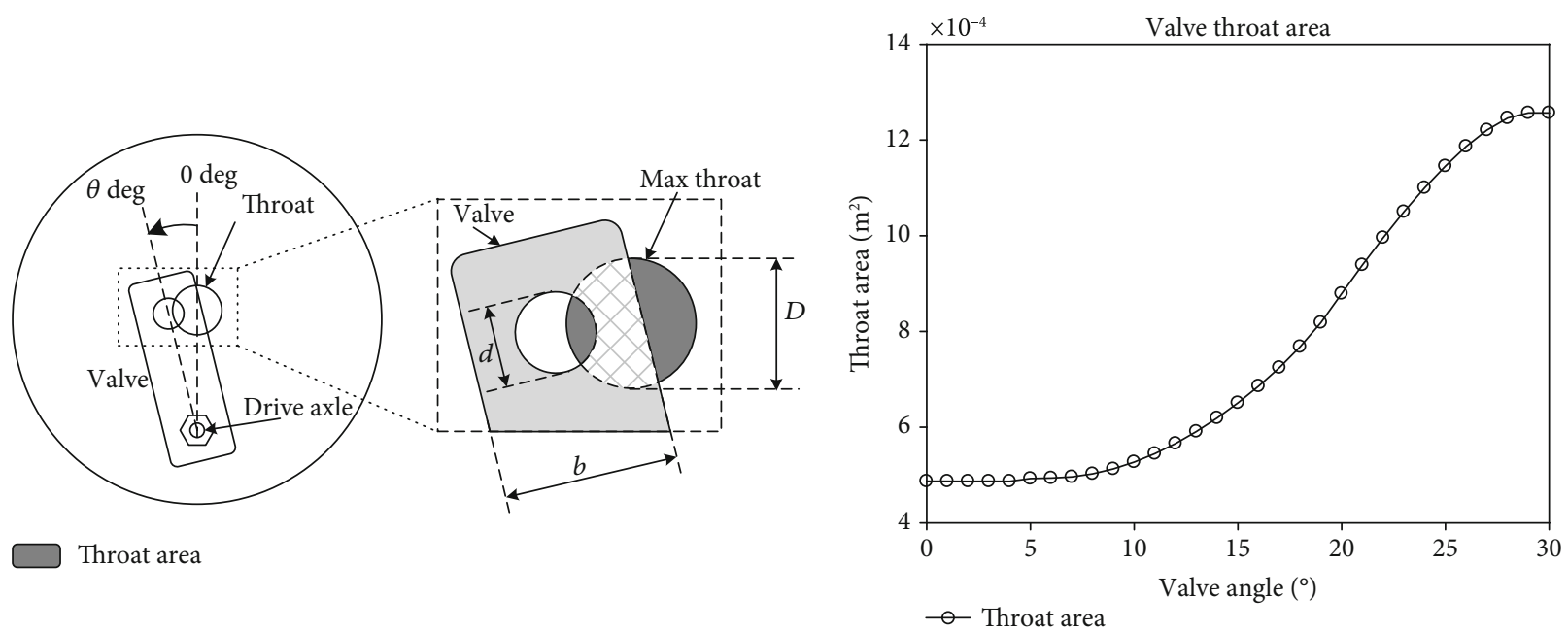

Figure 3: The platform of the gas generator and schematic of the throat area.

angle command as an input and valve angle as the output. The difference between the input and the output is defined as the backlash of the gas generator system, and the size of the backlash is described by the symbol $\sigma$.

There are many nonlinear modeling methods for backlash, like the dead zone model, the hysteretic model, vibration, and the impulse model $[17,18]$. In the chapter below, the hysteretic model is used to describe and analyze the backlash in the gas generator. The input motor angle is defined as $\theta_{M}$, and the output valve angle is defined as $\theta_{V}$. Due to the backlash in the system, the input and output will not be consistent during the regulation process, and their relationship is shown in Figure 4.

Suppose that the backlash is half in both sides at the initial time when the motor is moving in the positive direction: the valve will not move at the beginning; it corresponds to segment $\mathrm{OB}$ in the diagram. As the motor continues to move, the backlash decreases in the motion direction, the mechanical face will touch, and the valve begins to move; the process corresponds to segment $\mathrm{BC}$ in the diagram. When the motor changes the moving direction to negative, the valve will not change to negative at once; the mechanical face should touch again in the negative direction to transfer the driving torque, and it corresponds to segment $\mathrm{CD}$ in the diagram. When the mechanical interface touches again, the valve begins to move to negative; it corresponds to segment DF in the diagram. The mathematical description of the relation above can be written as follows:

$\theta_{V}= \begin{cases}m\left[\theta_{m}(t)-\sigma / 2\right], & \dot{\theta}_{v}(t)>0 \cap \theta_{v}\left(t^{-}\right)=m\left[\theta_{m}\left(t^{-}\right)-\sigma / 2\right], \\ \theta_{m}\left(t^{-}\right), & \text {otherwise, } \\ m\left[\theta_{m}(t)+\sigma / 2\right], & \dot{\theta}_{v}(t)<0 \cap \theta_{v}\left(t^{-}\right)=m\left[\theta_{m}\left(t^{-}\right)+\sigma / 2\right],\end{cases}$

where $t^{-}$is the state in the last time moment, $m$ is the proportionality coefficient of $\theta_{M}$ and $\theta_{V}$, it is also the slope of the oblique line $\mathrm{AC}$ and DF in Figure 4, and the value of $m$ is equal to 1 in the gas generator.

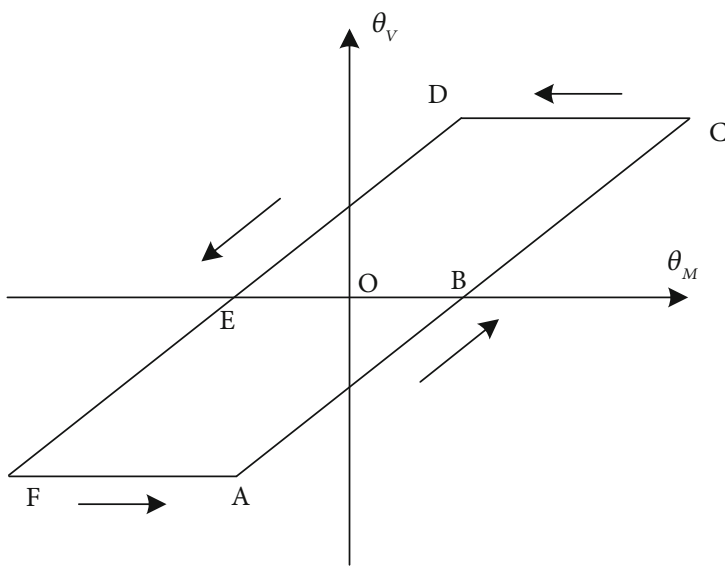

FIGURE 4: Hysteretic model of backlash.

There is an assumption for this hysteresis model: when the backlash is in the hold stage CD and FA, the output of the driven part (valve) can keep constant, and its underlying cause is that there is an existing large damping or load in the driven part to keep the valve still during the hold stage. The load characteristics for the flow regulator of a solid ducted rocket are discussed in reference [19]. It is obvious that the valve has a large system load and working load to keep it still during the hold stage; in other words, the assumption for the hysteresis model stands.

2.3. Influence on the System Control. Backlash is a ubiquitous phenomenon in an electromechanical system, and its influence on gas flow regulation and system control can be described in Figure 5.

The mechanical clearance between the motor and the valve must be eliminated in the motion direction to move the valve. When the motion direction changes, the mechanical clearance must be eliminated again. The process of elimination causes the response hysteresis of the valve angle; in view of the system control, it will cause the hysteresis of gas flow, and the influence is shown with a simulation result in Figure 6. 


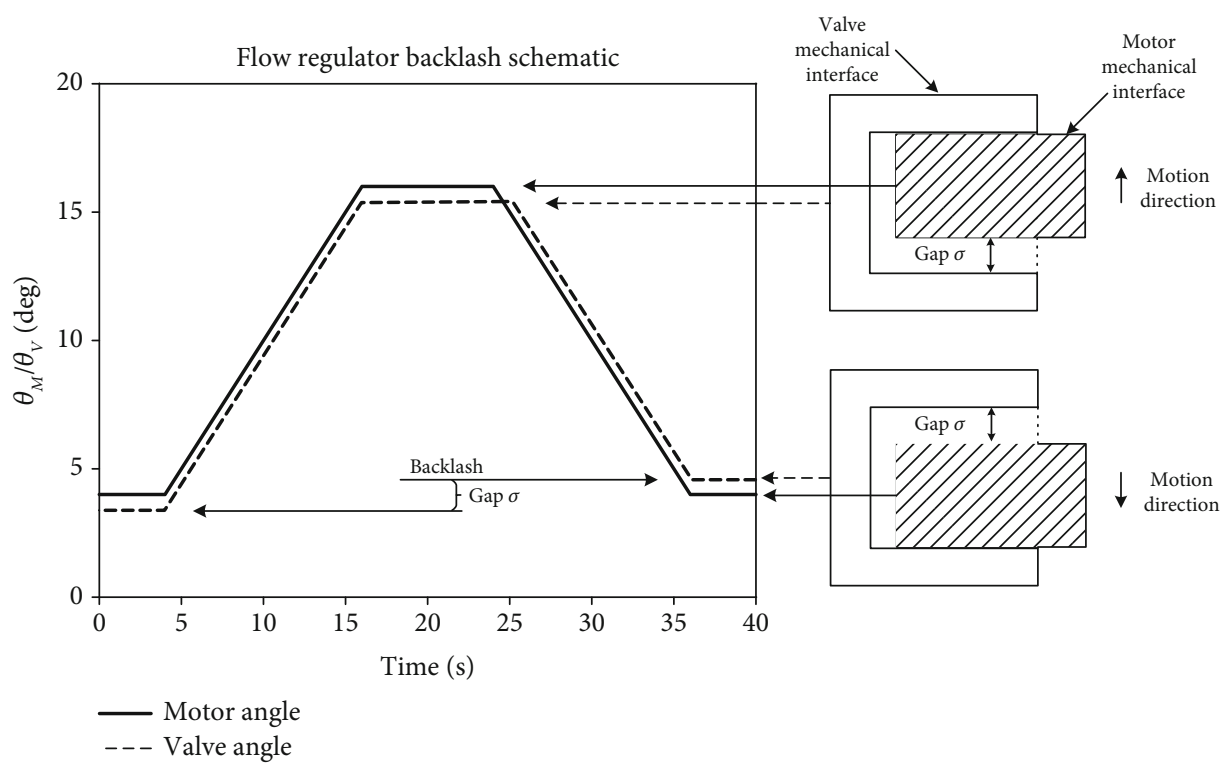

FIGURE 5: Schematic representation of flow regulator backlash.
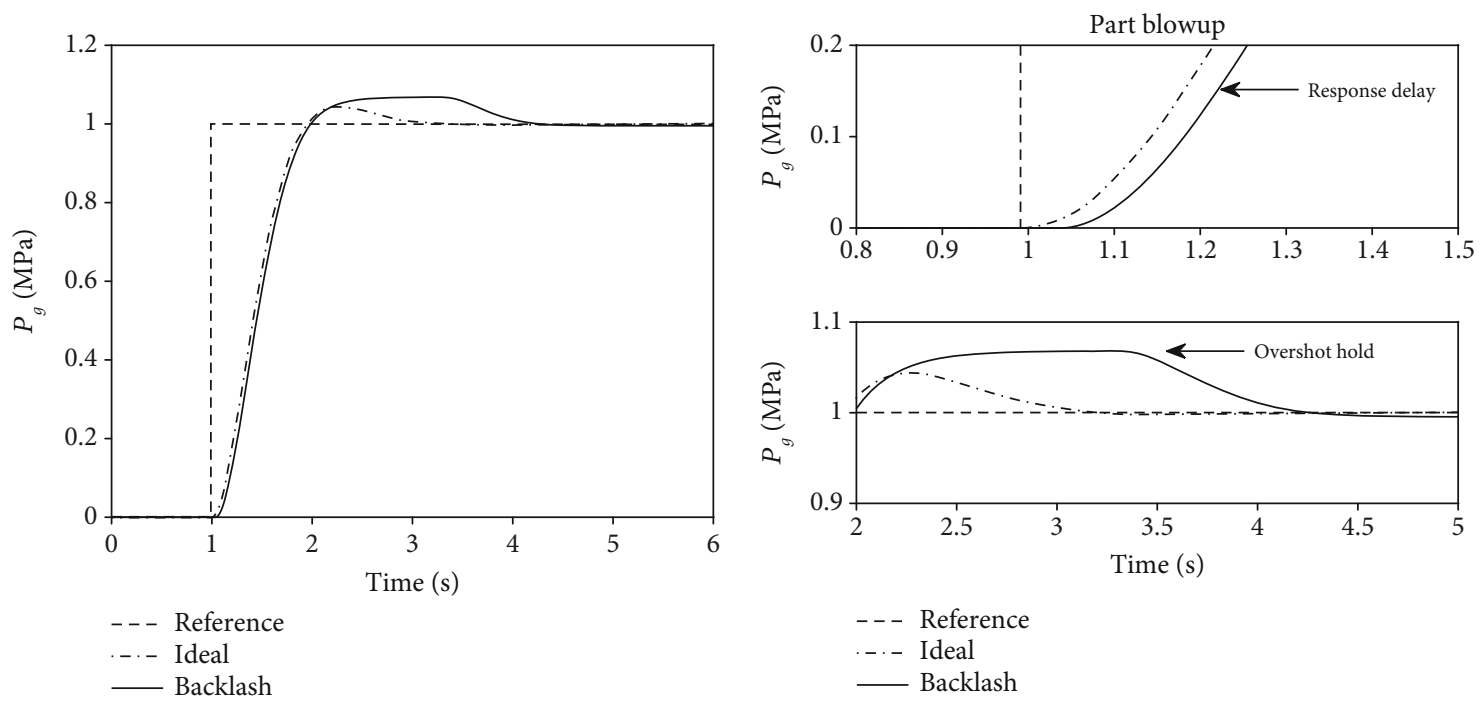

FIGURE 6: Effects of backlash on the pressure control system.

The simulation case compares the underdamped closedloop system response results in ideal conditions and backlash conditions. At the beginning of regulation, the response of the backlash case will have a little hysteresis as shown in Figure $6(t=1 \mathrm{~s})$, and it corresponds to segment $\mathrm{OB}$ in Figure 4. After that, when an overshoot happens $(t=2 \mathrm{~s})$, the response of the ideal case will turn back to the steady state at once. However, the backlash cases will remain at the overshoot state for a while $(t=2.2 \sim 3.4 \mathrm{~s})$, and the process of maintenance corresponds to segment CD in Figure 4.

In a general way, an underdamped closed-loop system responds faster than an overdamped system for the same plant. However, the underdamped system cannot achieve the ideal adjustment effect in Figure 6, due to the nonlinear part generated by the backlash, and this phenomenon introduces a delay to the control system and increases the adjust- ment time. In response, some control systems will be designed as an underdamped system to avoid the hysteresis when the overshoot happens. However, an underdamped system will decrease the response speed and will not be able to solve the hysteresis fundamentally; the hold stage CD and FA must go through when the demand gas flow turns to an opposite trend and the hysteresis cannot be avoided in these cases. A more effective method should be explored to fundamentally solve the hysteresis caused by the backlash.

\section{Touch State and Backlash Compensation Principle}

3.1. Touch State. The influence of a backlash on gas flow regulation and system control is described in Section 2.3 because of a nonlinear hysteresis element in the system. A linear 

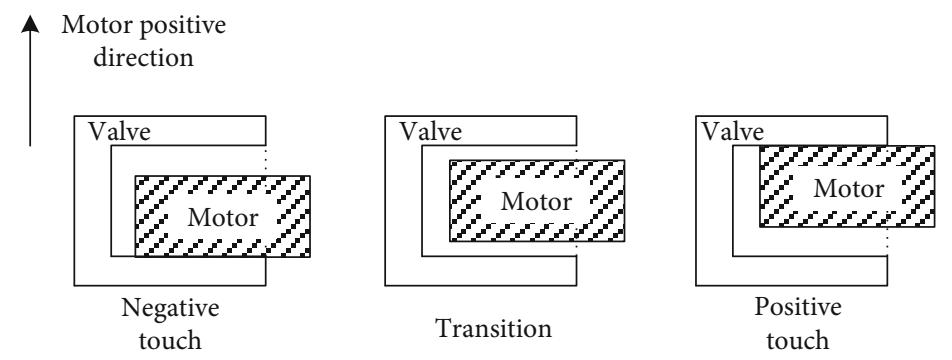

FIgURE 7: Schematic of backlash touch state.

method can hardly meet the challenge of a backlash, and so, here we analyze a nonlinear compensation method. From the influence principle of a backlash, the key point of the backlash compensation method lies in the state observation of a backlash, which includes the size of the backlash and its existing direction.

According to the demand above, the backlash can be divided into three states, and it is defined as the touch state $T_{f}$ to describe the state of a backlash:

$$
T_{f}= \begin{cases}-1, & \text { negative touch } \\ 0, & \text { transition, } \\ 1, & \text { positive touch }\end{cases}
$$

Firstly, we defined the positive direction of the motor as the positive direction of the touch state; when the mechanical face of the motor touches the mechanical face of the valve in a positive direction, this state is defined as positive touch and it is shown in the right part of Figure 7. When the two mechanical faces touch in the negative direction, this state is defined as the negative touch and it is shown in the left part of Figure 7. If the two mechanical faces do not touch at any side, it is defined as the transition state and is shown in the middle part of Figure 7; it is obvious that the transition state is the major cause of the response hysteresis in the flow regulation.

The definition of the touch state can be used to estimate the state of the backlash, including the existing direction of the backlash, and whether the mechanical face touches or not, it can provide significant information for the backlash compensation.

3.2. Touch State Update. The touch state can be used to evaluate the backlash, and its update law is the key to observe the backlash state and apply it to real-time control. The two update rules of the touch state are analyzed in the following text.

As is analyzed above, when the touch state is not a transition state, the touch state will only change when the motor changes its motion direction; the same direction of motion or remaining motionless will not change the touch state. Thus, it can be seen that the motion intention is a decision condition for the update of the touch state, and the motion intention
$M_{f}$ can be defined based on the motor command or the controller command:

$$
M_{f}= \begin{cases}-1, & \theta_{c}(t)-\theta_{c}\left(t^{-}\right)<0, \\ 0, & \theta_{c}(t)-\theta_{c}\left(t^{-}\right)=0, \\ 1, & \theta_{c}(t)-\theta_{c}\left(t^{-}\right)>0,\end{cases}
$$

where $\theta_{c}\left(t^{-}\right)$is the controller command at the last time moment.

In a real control system, the response speed of an actuator cannot be infinitely high; in other words, when the touch state changes, a negative touch state cannot switch to a positive touch state directly, and a transition state is a necessary process between the two states.

Based on the description above, a positive motion intention will change a negative touch state into a transition state; then, it will change into a positive touch state if the positive motion intention is kept. Similarly, a negative motion intention will change a positive touch state into a transition state; then, it will change into a negative touch state if the negative motion intention is kept. That is the first rule of the touch state update.

A positive or negative touch state that changes into a transition state can be determined by the motion intention; however, in order to confirm when a transition state changes into a positive or negative touch state, we need other information. As shown in Figure 3, the throat area increases with the increase of the valve angle. According to the working principle of a chocked ducted rocket, the pressure of a gas generator will decrease with the increase of the throat area; that is to say, the motion of a valve angle will be reflected in the change of the pressure, and its rule is clear. The gate valve in the gas generator is an active part to regulate the gas flow by changing the pressure. If the pressure is changing from a stable state, it is certain that the valve is driven by the motor and the two mechanical faces touch one side; in other words, the touch state of a backlash cannot be the transition state in these cases, and whether it is a positive touch state or a negative touch state depends on whether the pressure decreases or increases. That is the second rule of the touch state update.

The two update rules of a touch state can be summarized by a diagrammatic representation in Figure 8 .

It is a sufficient but not a necessary condition to deduce the touch state from the change of pressure in the previous 


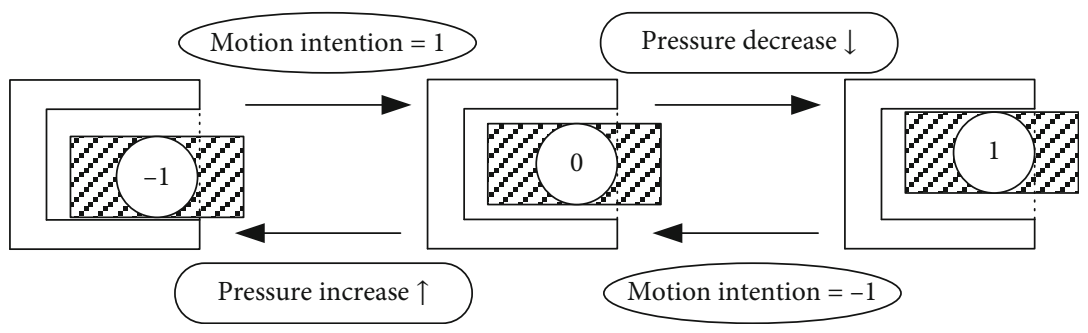

FIGURE 8: Schematic of a backlash touch state update.

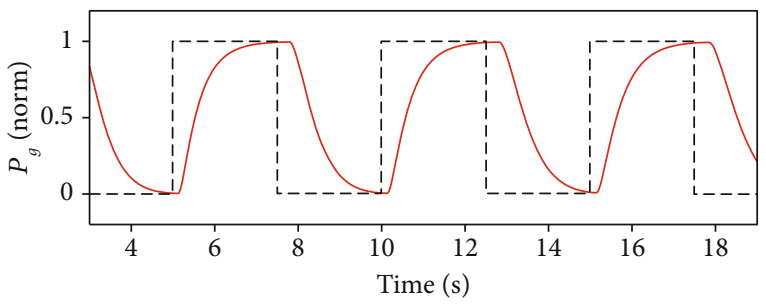

- - - Reference $-P_{g}$

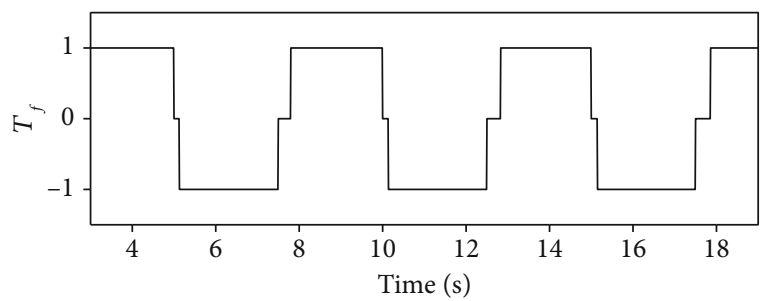

FIGURE 9: Observation of touch state in a flow regulation.

description; it means that the change of pressure can be used to deduce the touch state, but it is not enough in return. The second rule of the touch state update used only the first half deduction, and it is strictly logical.

Based on the theoretical analysis above, the mathematical description of the touch state update can be written as follows:

$T_{f}(t)= \begin{cases}-1, & T_{f}\left(t^{-}\right)=0 \cap P_{g}(t)-P_{g}\left(t^{-}\right)>0, \\ 0, & {\left[T_{f}\left(t^{-}\right)=-1 \cap M_{f}(t)=1\right] \cup\left[T_{f}\left(t^{-}\right)=1 \cap M_{f}(t)=-1\right],} \\ 1, & T_{f}\left(t^{-}\right)=0 \cap P_{g}(t)-P_{g}\left(t^{-}\right)<0,\end{cases}$

where $T_{f}\left(t^{-}\right)$and $P_{g}\left(t^{-}\right)$are the touch state and gas generator pressure at the last time moment, respectively, and $M_{f}(t)$ is the motion intention defined in equation (3); the touch state of a backlash can be observed in real time and a simulation result is proposed in Figure 9.

From the simulation results above, the transition touch state $\left(T_{f}=0\right)$ is consistent with the hysteresis stage of response on the timeline. In essence, the hysteresis is the motor motion that has not been transferred to the valve due to the backlash, and the hysteresis will not end until the backlash is eliminated in the motion direction. For a con-

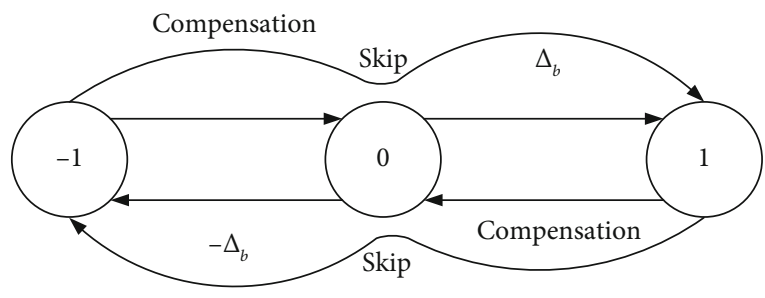

FIGURE 10: Schematic of touch state switch accelerate.

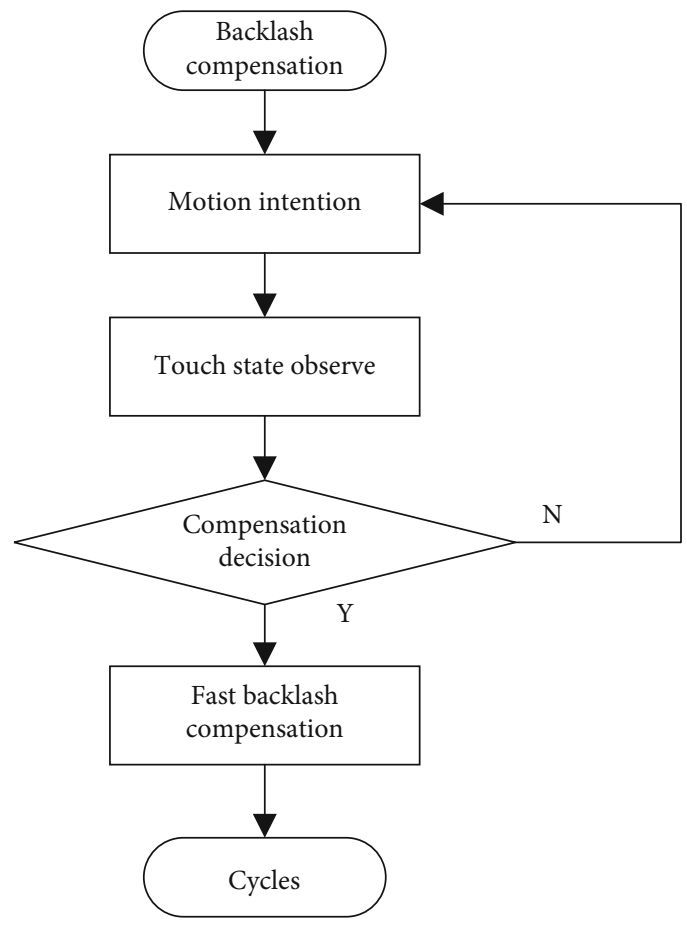

FIGURE 11: Process diagram of backlash compensation.

stant backlash, the duration time of the hysteresis stage is related to the regulation speed: the faster the motor motion is, the earlier the hysteresis will end. The gas generator is a typical variation parameter system; an adaptive controller will change the controller parameter according to the system state, and a bigger controller parameter will end the hysteresis faster if the system does not consider the backlash during its controller design. On the other hand, the backlash influence will expand in some conditions. This also explains why the duration of a transition touch state is not the same as the whole regulation process as shown in Figure 9. 

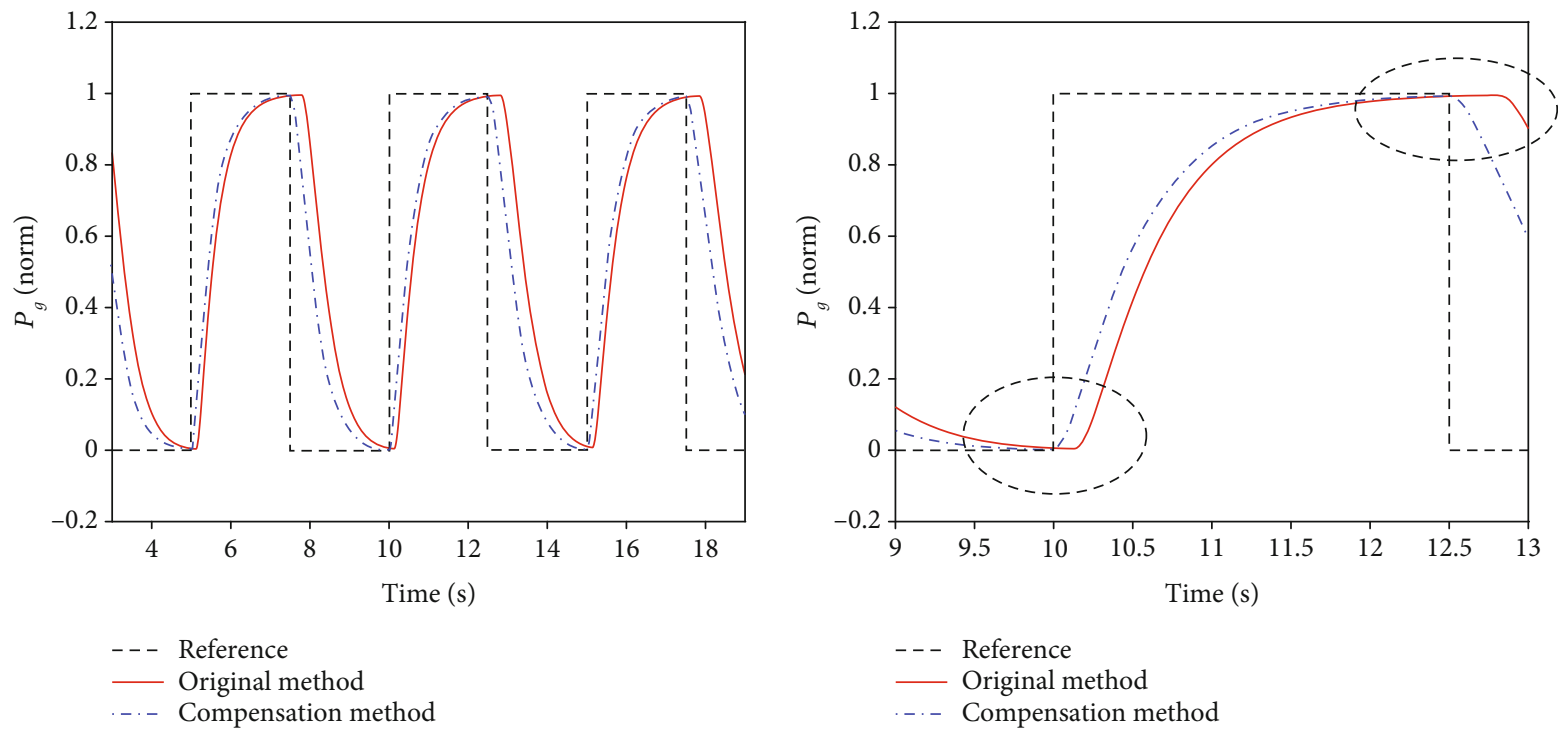

FIGURE 12: Simulation result of backlash compensation in a constant case.

3.3. Principle of Backlash Compensation. The transition touch state is the root cause of the hysteresis. If the negative touch state can switch to a positive touch state as soon as possible, the duration time of hysteresis can be greatly reduced; so does the influence of backlash. Using the touch state observation, we observe the moment it is clear that the negative touch state changes to the transition state, and the feedback circuit can be improved by adding a compensation angle at that moment to accelerate the process of transition.

For example, when a negative touch state $\left(T_{f}=-1\right)$ is changing into a positive touch state $\left(T_{f}=1\right)$ under the action of the control system, a compensation angle $\Delta_{b}$ will help the touch state skip the transition state as soon as possible. And the compensation angle $\Delta_{b}$ is equal to the size of the backlash that is defined in Section 2.2 The process that the positive touch state $\left(T_{f}=1\right)$ changes into a negative touch state $\left(T_{f}=-1\right)$ can be accelerated in the same way, and the backlash compensation principle can be summarized by a diagrammatic representation shown in Figure 10.

The backlash compensation of a backlash can be separated into three steps: firstly, get the latest state of motion intention and touch state in every control period. Then, the compensation decision must be made according to the two states in step one. Finally, if the decision above is a "yes," a compensation angle will be added in the control loop to skip the transition state of the backlash; otherwise, the compensation will not step in. The three-step process is shown in Figure 11 .

\section{Backlash Compensation Method and Verification}

Here, we propose the backlash compensation method and its verification, and it is carried out under two situations, namely, that the size of the backlash is known and yet unknown.

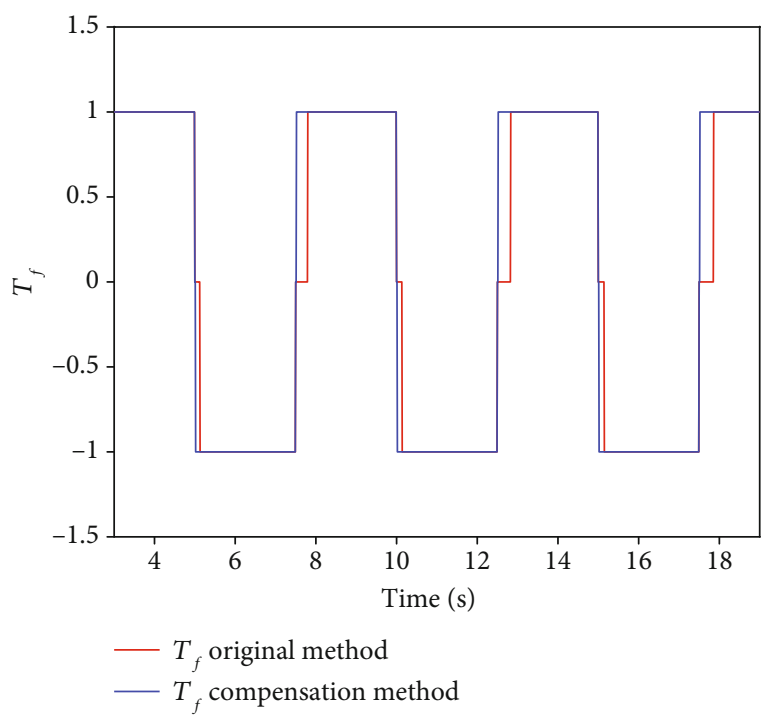

Figure 13: Compare results of backlash touch state.

4.1. A Constant Backlash Compensation Method. The backlash compensation control method is an upgrade based on an integral controller, and the control law of the original integral controller in a discrete format can be written as follows:

$$
\theta_{c}(k)=\theta_{c}(k-1)+K_{I}\left(P_{g 0}, V_{0}\right) \cdot d T \cdot d P_{g},
$$

where $K_{I}\left(P_{g 0}, V_{0}\right)$ is the controller parameter which varies with the pressure and free volume of the gas generator, $d T$ is the discrete-time intervals, $d P_{g}$ is the difference of the pressure command and feedback. The new control law adding backlash compensation function can be written as follows:

$$
\theta_{c}(k)=\theta_{c}(k-1)+K_{I}\left(P_{g 0}, V_{0}\right) \cdot d T \cdot d P_{g}+\Delta_{b}
$$



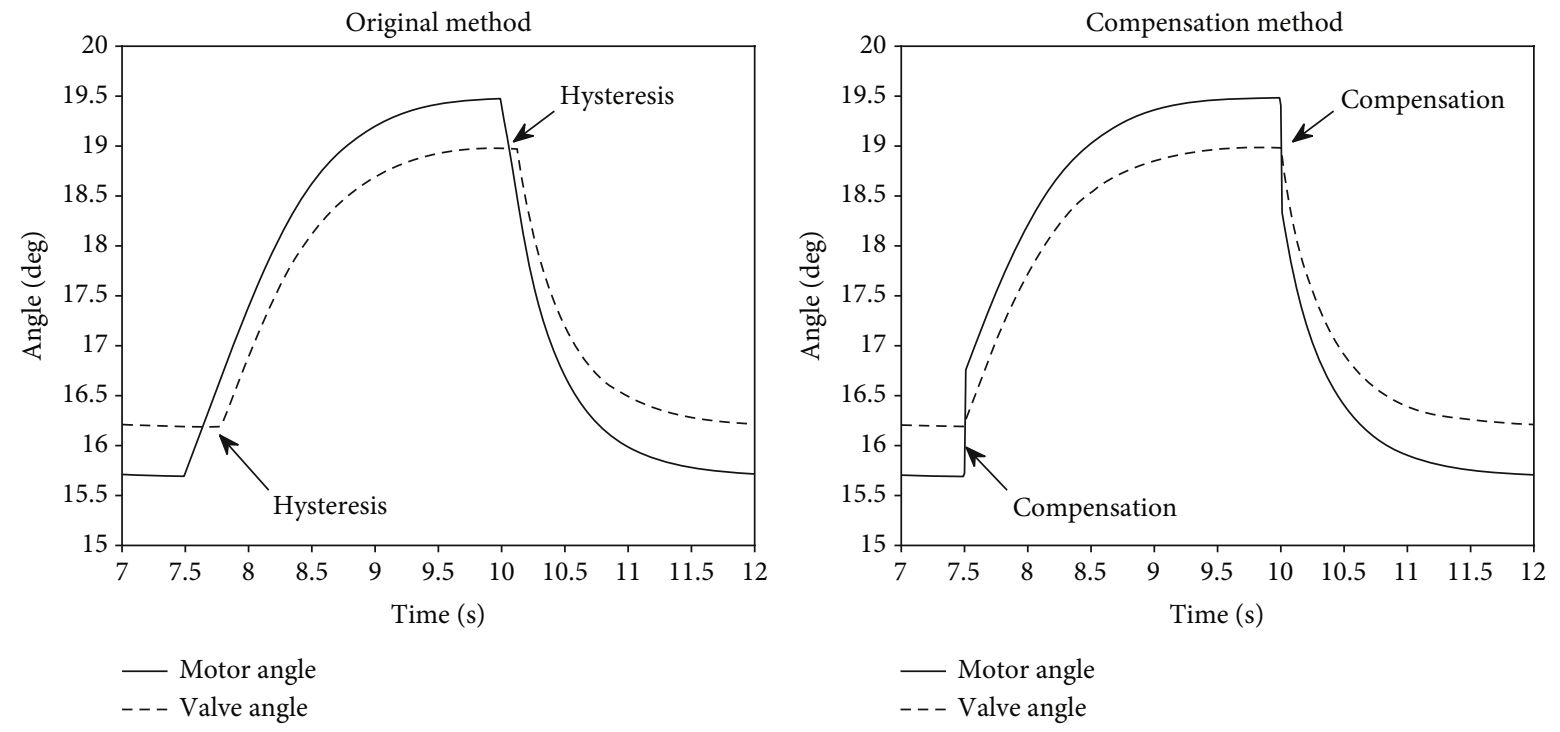

FIGURE 14: Result of motor angle and valve angle by a constant compensation method.

where $\Delta_{b}$ is the backlash compensation value, and its definition is as follows:

$$
\Delta_{b}= \begin{cases}-\sigma, & T_{f}(k-1)=1 \cap T_{f}(k)=0, \\ \sigma, & T_{f}(k-1)=-1 \cap T_{f}(k)=0, \\ 0, & \text { otherwise. }\end{cases}
$$

The backlash compensation value is equal to the size of the backlash when the compensation is needed. The cases include negative touch state change into a transition state and positive touch state change into a transition state; otherwise, the compensation value is zero. Suppose that the size of the backlash is known and constant during the regulation; there are many ways to measure the size of a backlash before the rocket works. The simulation result with a known backlash is carried out in Figure 12, and the size of a backlash is set as $\sigma=1$ degree.

According to the compared result of the backlash compensation method and the original control method, the backlash compensation method can indeed accelerate the response, and the hysteresis caused by the backlash is eliminated. The compared result of the touch state is also shown in Figure 13.

The touch state observation result of the compensation method is different from the original control method. Its difference lies in the transition state: the duration time of the transition state in the compensation case is extremely short, and the transfer from the positive touch state to the negative touch state is smooth and anhysteretic; so does the transfer from the negative touch state to the positive touch state.

The inconsistent valve angle and motor angle are the root causes of response hysteresis, and the motor angle and valve angle are compared in Figure 14.

The valve and motor angles of the original control method are shown in Figure 14(a). At the transition state (time: $7.5 \mathrm{~s} \sim 7.8 \mathrm{~s}$ ), the motion of the motor did not transfer to the valve, and it keeps still. However, at the same regulation situation for the backlash compensation method, a compensation angle was added at that moment, and the valve moved with the motion of the motor without any hysteresis, as shown in Figure 14(b). The situation above is a negative touch state that transfers to a positive touch state, and the situation that a positive touch state transfers to a negative touch state are also shown in Figure 14 at time 10 s; the principle is the same.

4.2. An Adaptive Backlash Compensation Method. In a real working process of a ducted rocket, the size of a backlash is not constant; it will change under the influence of heat and stress as the gas generator is working, and the compensation method for a constant and known backlash that is proposed in Section 4.1 will not suitable for this situation.

An adaptive compensation method is proposed and is aimed at the variation of the backlash. It is upgraded based on the constant compensation method; its advancement is embodied in the adaptive law of the compensation angle. The format of the control law is the same as the constant compensation method:

$$
\theta_{c}(k)=\theta_{c}(k-1)+K_{I}\left(P_{g 0}, V_{0}\right) \cdot d T \cdot d P_{g}+\Delta_{b}
$$

where $\Delta_{b}$ is the backlash compensation value, and its definition is as follows:

$$
\Delta_{b}= \begin{cases}-\sigma_{r}, & T_{f}\left(t^{-}\right)=1 \cap T_{f}(t)=0, \\ \sigma_{r}, & T_{f}\left(t^{-}\right)=-1 \cap T_{f}(t)=0, \\ 0, & \text { otherwise, }\end{cases}
$$

where $\sigma_{r}$ is not a constant valve as $\sigma$ in equation (7). The compensation value will adjust according to the response 


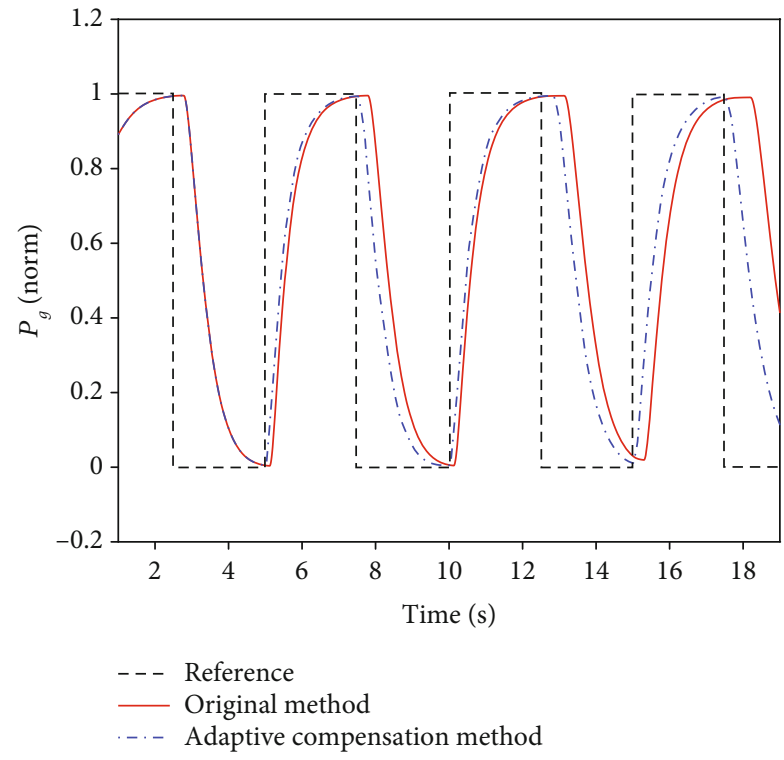

FIgURE 15: Simulation results of backlash compensation in an adaptive case.

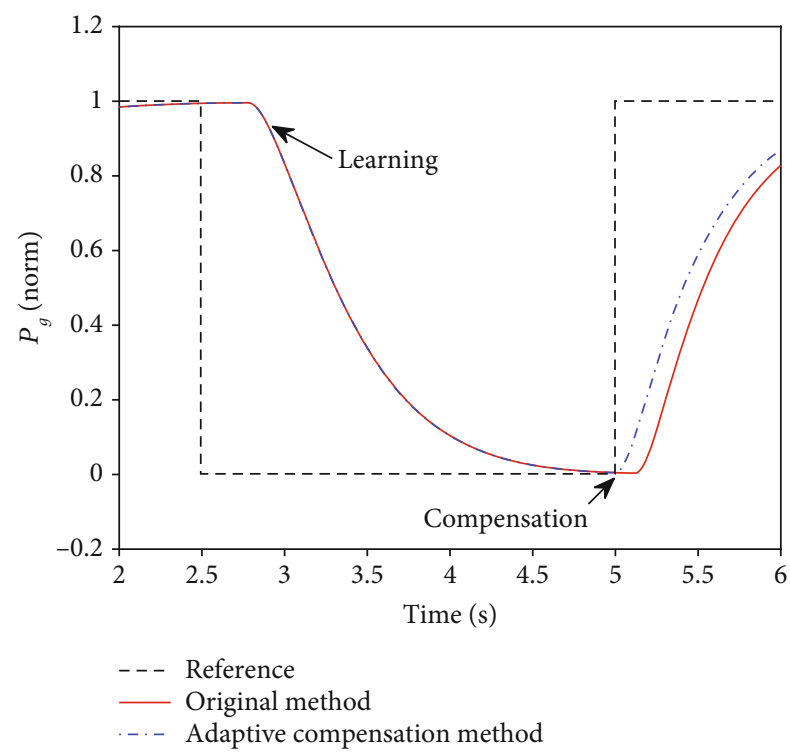

FIgURE 16: Simulation result of backlash compensation in an adaptive case (part I blowup).

hysteresis during the regulation, and the adaptive law of compensation value is designed as follows:

$$
\sigma_{r}(k)=\sigma_{r}(k-1)+\left|K_{I}\left(P_{g 0}, V_{0}\right) \cdot d T \cdot d P_{g}\right| \cdot\left(1-\left|T_{f}(k)\right|\right) .
$$

The controller output during the transition is the difference between the actual size of the backlash and the present compensation value. Then, the controller output during the transition state will be recorded and accumulated into the compensation value to eliminate the difference, and the new compensation value will be applied in the next touch state transfer.

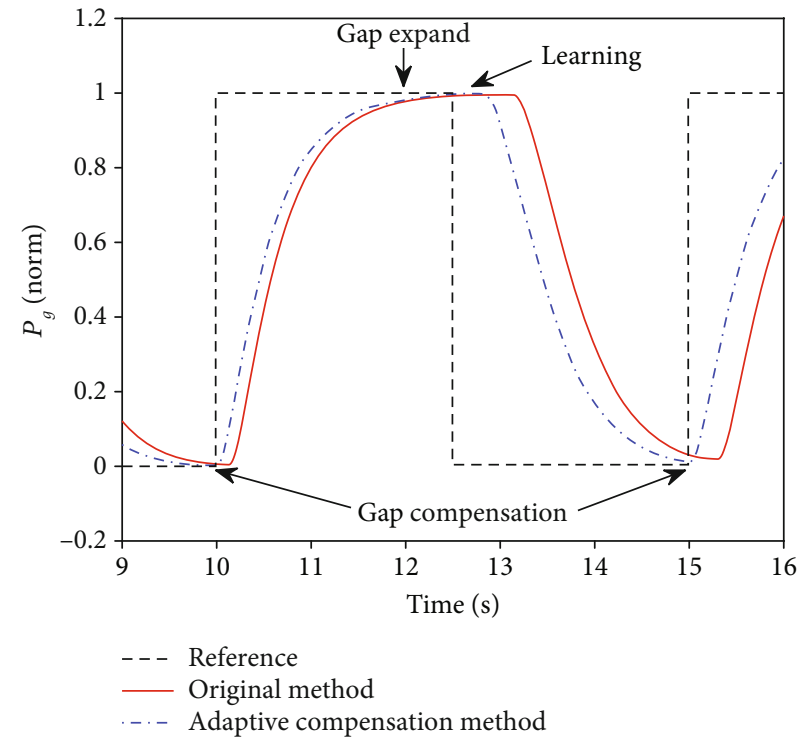

FIGURE 17: Simulation results of backlash compensation in an adaptive case (part II blowup).

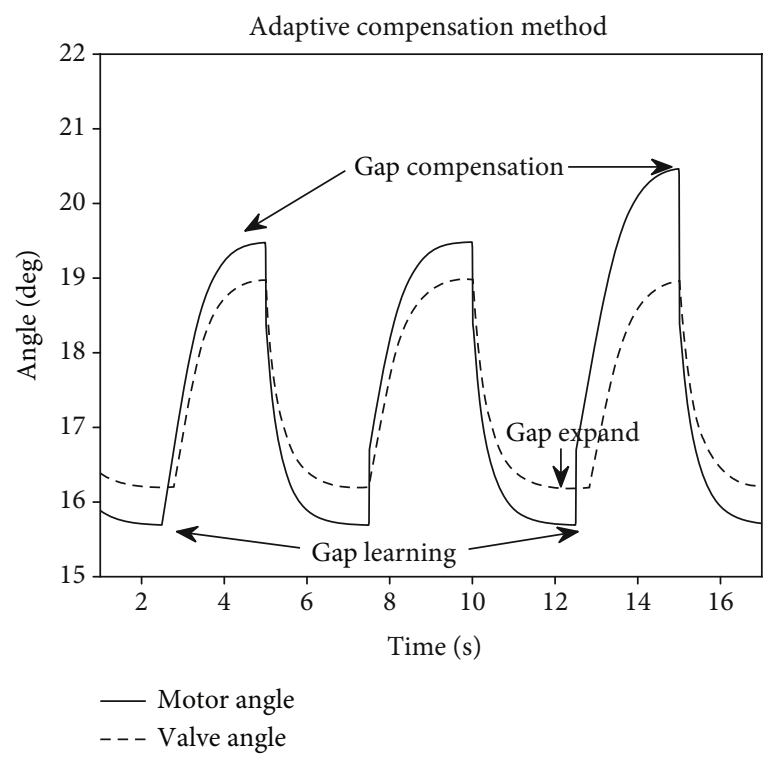

FIgURE 18: Result of the motor angle and the valve angle by an adaptive compensation method.

A simulation was carried out to verify the adaptive method: suppose the size of backlash was not measured and is unknown before the system works, and suppose the size of backlash will expand under the influence of heat and stress. The simulation initialization sets the size of the backlash at 1 degree, and it will expand to 2 degrees at time $12 \mathrm{~s}$. Figure 15 shows the results.

The first regulation is at time $2.5 \mathrm{~s}$. Because the size of the backlash is unknown before the system works, the responses of the compensation method and the original method are the same at the first time, but the adaptive compensation method is at a learning state to get the size of the backlash at this time. When it comes to the second regulation, the time is at $5 \mathrm{~s}$. The difference between the compensation method and the 
original method emerges, and the compensation method response with no hysteresis is shown in Figure 16.

The expansion of the backlash is set at the time $12 \mathrm{~s}$ in the simulation. Before the expansion, the regulation result of the compensation method is good, but the response hysteresis reappears at the next regulation at time $12.5 \mathrm{~s}$ due to backlash expansion. At that time, the difference of the backlash is recorded by the method, the adjustment of compensation angle is made, and the hysteresis is eliminated again at time $15 \mathrm{~s}$, as shown in Figure 17.

A comparison of the result of the compensation method and the original method is also shown in the difference of the motor angle and the valve angle in Figure 18.

The compensation method is in a learning state, and the valve angle has hysteresis at time $2.5 \mathrm{~s}$. The compensation angle can be seen in the following regulation, the backlash was compensated by the added motor angle, and the valve can move without hysteresis. After the backlash expansion happens at time $12.5 \mathrm{~s}$, the compensation angle that adjusted by the previous regulation is not enough, and the hysteresis reappears, although a compensation angle is added to the motion of the motor. However, in the following regulation, after the adjustment, a bigger compensation angle is added, and the hysteresis of the valve is eliminated.

From the results above, the adaptive backlash compensation method can deal with the influence of the backlash very well, especially in situations where the size of the backlash is unknown and is changing while being regulated.

\section{Conclusions}

A research of the backlash of the flow regulator in a solid ducted rocket was carried out in this paper, a hysteresis model was built to describe the backlash, and its influence on the engine control was analyzed. An adaptive backlash compensation method was proposed under the challenge of limited information caused by the limit of the sensor in an aircraft and the challenge of backlash state variation caused by the harsh environment in the gas generator. The touch state of a backlash is designed, and its observation using existing information is carried out to get the state of the backlash, including its size and existing direction. Then, a compensation control method combined with the motion intention is proposed. This method greatly shortened the time during the transition and reduced the hysteresis effect on the engine control system. Furthermore, the method keeps improving, as the compensation parameter can change adaptively according to the response deviation during the process of flow regulation, and it can meet the challenge where the state of the backlash is unknown or variable. Finally, the validation of the adaptive backlash compensation method was carried out with two simulations.

The compensation method in this work will hopefully solve the backlash problem in a real ducted rocket, but further work is needed to improve this method. In order to prove the robustness of this method, further work needs to be done, and reliable hardware in a loop simulation is needed before applying it to a real ducted rocket test. To extend the application of this method, different types of the throttling valve can be attempted in further research.

\section{Data Availability}

The data used to support the findings of this study are available from the corresponding author upon request.

\section{Conflicts of Interest}

The authors declare no conflict of interest.

\section{Acknowledgments}

This work was supported by the National Natural Science Foundation of China (Grant No. 61174120); Scientific Research Starting Foundation for the Introduced Talents, Sun Yat-sen University.

\section{References}

[1] D. Huo, D. Yan, and B. Gao, "Research progresses and prospect of variable flow ducted rocket technologies," Journal of Solid Rocket Technology, vol. 40, no. 1, pp. 11-19, 2017.

[2] H. Besser, "History of ducted rocket development at Bayern Chemie," in 44th AIAA/ASME/SAE/ASEE Joint Propulsion Conference \& Exhibit, pp. 21-23, Hartford, CT, USA, July 2008.

[3] Z. Lv, Z. Xia, and B. Liu, "Review of research on solid fuel scramjet engine," Journal of Aerospace Power, vol. 31, no. 8, 2016.

[4] R. A. Stowe, C. Dubois, P. G. Harris, A. E. H. J. Mayer, A. DeChamplain, and S. Ringuette, "Performance prediction of a ducted rocket combustor using a simulated solid fuel," Journal of Propulsion and Power, vol. 20, no. 5, pp. 936-944, 2004.

[5] F. Lan, L. Wang, and X. Chen, "A flow controller design for solid-rocket ramjet motor," Journal of Projectiles, Rockets, Missiles and Guidance, vol. 32, no. 3, pp. 148-151, 2012.

[6] H. Sun and X. Wang, "Numerical simulation of solid rocket ramjet performance by using flow regulation valve," Aeronautical Science and Technology, vol. 3, pp. 52-54, 2011.

[7] J. Chang, B. Li, and W. Bao, "Friction-compensation control of gas-flow regulation for ducted rockets based on adaptive dither method," Journal of Aerospace Engineering, vol. 26, no. 4, pp. 715-720, 2013.

[8] L. Ma, Z. Xia, and J. Hu, "Effects of valve velocity on the dynamic response characteristics of variable flow ducted solid rocket motor," Missiles and Space Vehicles, vol. 2, pp. 8-13, 2012.

[9] A. Szmidt and W. Gierulski, "Identification of a backlash using the modeling function method," Agh University of ence \& Technology Press, vol. 24, no. 2, pp. 150-154, 2005.

[10] Z. Xu, Q. Jing, and J. Wang, "Control system and method of reversing backlash compensation for high precision mechanical equipment," Heavy Machinery, vol. 2, pp. 13-16, 2018.

[11] S. Jiang, F. Tian, and W. Liang, "Backlash compensation method in naval gun servo system based on global backstepping sliding mode control," Journal of Naval University of Engineering, vol. 30, no. 5, pp. 92-98, 2018. 
[12] Y. Shota and F. Hiroshi, "Precise joint torque control method for two-inertia system with backlash using load-side encoder," IEEJ Journal of Industry Applications, vol. 8, no. 1, pp. 73-75, 2019.

[13] Y. Chang, Y. Wang, F. E. Alsaadi, and G. Zong, "Adaptive fuzzy output-feedback tracking control for switched stochastic pure-feedback nonlinear systems," International Journal of Adaptive Control and Signal Processing, vol. 33, no. 10, pp. 1567-1582, 2019.

[14] J. Mao, Z. Xiang, and S. Huang, "Adaptive finite-time tracking control for a class of switched nonlinear systems with unmodeled dynamics," Neurocomputing, vol. 196, pp. 42-52, 2016.

[15] J. I. Xiao-Zheng, Y. A. Guang-Hong, C. XH, and C. H. WeiWei, "Robust Fault-tolerant Ho Control with Adaptive Compensation," Acta Automatica Sinica, vol. 39, no. 1, pp. 31-42, 2013.

[16] W. Miller, W. Burkes, and S. Mcclendon, "Design approaches for variable flow ducted rockets," in AIAA/SAE/SAME 17th Joint Propulsion Conference, pp. 1981-1989, Colorado, USA, 1981.

[17] G. Ma, Z. Wang, and F. Wang, "Friction measurement, identification, and compensation for servomechanisms," in 2011 International Conference in Electrics, Communication and Automatic Control Proceedings, pp. 1469-1475, Chongqing, China, 2011.

[18] J. Lu, H. Chen, X. Sun, and Y. Xu, "Dynamic modeling and analysis of robot arm with consideration of clearance in gear reducer," Journal of Mechanical Engineering, vol. 49, no. 15, pp. 15-21, 2013.

[19] A. Wang and Q. Zeng, "Load characteristics and modeling methods for the flow regulator of a solid ducted rocket," International Journal of Aerospace Engineering, vol. 2019, Article ID 8031290, 10 pages, 2019. 